Research Paper

\title{
Survival Benefits of Small Anatomical Resection of the Liver for Patients with Hepatocellular Carcinoma and Impaired Liver Function, Based on New-Era Imaging Studies
}

\author{
Masahiko Sakoda ${ }^{1 凶}$, Shinichi Ueno ${ }^{2}$, Satoshi Iino ${ }^{1}, K$ iyokazu Hiwatashi ${ }^{1}$, Koji Minami $^{1}$, Yota Kawasaki ${ }^{1}$, \\ Hiroshi Kurahara ${ }^{1}$, Yuko Mataki ${ }^{1}$, Kosei Maemura ${ }^{1}$, Hiroyuki Shinchi ${ }^{3}$, Shoji Natsugoe ${ }^{1}$
}

1. Department of Digestive Surgery, Breast and Thyroid Surgery, Kagoshima University School of Medicine, 8-35-1 Sakuragaoka, Kagoshima 890-8520, Japan.

2. Department of Clinical Oncology, Kagoshima University School of Medicine, 8-35-1 Sakuragaoka, Kagoshima 890-8520, Japan.

3. Kagoshima University Graduate School of Health Sciences, 8-35-1 Sakuragaoka, Kagoshima 890-8520, Japan.

$\square$ Corresponding author: Masahiko Sakoda, MD, PhD, Department of Digestive Surgery, Breast and Thyroid Surgery, Kagoshima University School of Medicine, 8-35-1 Sakuragaoka, Kagoshima 890-8520, Japan. Tel: 81-99-275-5361; Fax: 81-99-265-7426; E-mail: sakoda@m.kufm.kagoshima-u.ac.jp

(1) Ivyspring International Publisher. Reproduction is permitted for personal, noncommercial use, provided that the article is in whole, unmodified, and properly cited. See http://ivyspring.com/terms for terms and conditions.

Received: 2016.02.02; Accepted: 2016.04.26; Published: 2016.05.25

\begin{abstract}
Background: It has been reported that anatomical resection of the liver may be preferred for primary hepatocellular carcinoma (HCC), and is at least recommended for systematic removal of a segment confined by tumor-bearing portal tributaries. However, nonanatomical resection (NAR) is often selected because of the patient's background, impairment of liver function, and tumor factors. The aims of the present study were to retrospectively compare the recurrence-free survival (RFS) rates for cases of partial resection (PR) and for small anatomical resection (SAR), which is regarded as NAR for primary HCC with impaired liver function.

Patients and Methods: So-called NAR was performed for a primary and solitary $(\leq 5 \mathrm{~cm}) \mathrm{HCC}$ in 47 patients; the patients were classified into PR $(n=25)$ and SAR $(n=22)$ groups. Clinicopathological factors, survival data, and recurrence patterns were compared between groups.

Results: There were no significant differences in the preoperative characteristics between the two groups. Operative time was significantly longer in the SAR group than in the PR group. There was no significant difference in the postoperative morbidity and tumor pathological characteristics between the two groups. The RFS of the SAR group was significantly better than those of the PR group. Although there was no significant difference in the pattern of recurrence between the two groups, the rate of intrahepatic recurrence in the same segment as the initial tumor tended to be higher in the PR group than in the SAR group. Multivariate analysis revealed that only the PR operative procedure was significant independent risk factor for poorer RFS.
\end{abstract}

Conclusion: Compared with PR, SAR effectively improves the rate of RFS after surgery for a primary and solitary HCC with impaired liver function.

Key words: hepatocellular carcinoma, anatomical resection, nonanatomical resection, hepatectomy, intrahepatic recurrence, liver cirrhosis

\section{Introduction}

It has been reported that anatomical resection (AR) of the liver may be preferred for primary hepatocellular carcinoma (HCC), and is at least

recommended for systematic removal of a segment confined by tumor-bearing portal tributaries [1-4]. We also reported that AR of a solitary HCC was superior 
to nonanatomical resection (NAR) or ablation therapies, in association with overall and disease-free survival [5]. However, NAR such as partial resection (PR) and enucleation is often also selected, with consideration of patient background variables, including comorbidity, the degree of impairment of liver function, tumor location, and extent of tumor spread.

"Subsegmentectomy" in Couinaud's classification [6] is regarded as NAR under Japanese general guidelines [7]. Couinaud's subsegment appears to comprise the liver parenchyma fed by the 4th order portal vein branch or by one of several 3rd order portal vein branches flowing into the same segment. Since 2006, we have aggressively employed resection of small anatomical divisions based on three-dimensional (3D) images as an alternative to PR for patients with impaired liver function. We identify the 3rd or 4th order portal vein territory feeding the domain using indocyanine green (ICG) or blue dye injection under intraoperative ultrasound (US) guidance, in order to improve the prognosis.

The aims of the present study were to retrospectively compare recurrence-free survival (RFS) rates for PR and small anatomical resection (SAR) for primary and solitary HCC of $5 \mathrm{~cm}$ or less with impaired liver function, and to elucidate the impact of SAR on recurrence after curative surgery, based on new imaging studies.

\section{Patients and methods}

\section{Study population}

A total of 158 patients with a primary and solitary HCC of $5 \mathrm{~cm}$ or less underwent curative

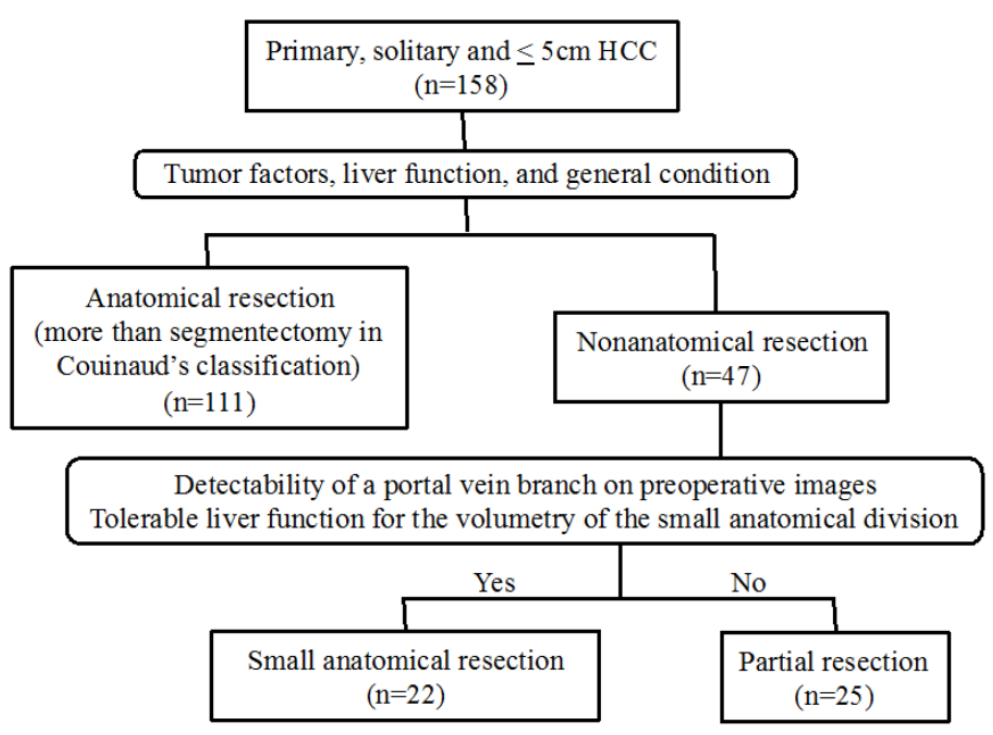

Figure 1. Flow diagram of therapeutic methods for patients with a primary, solitary and $\leq 5 \mathrm{~cm}$ HCC. hepatic resection in our department between January 2005 and December 2013. Among these, 111 patients underwent $\mathrm{AR}$, and 47 with impaired liver function or severe comorbidity underwent NAR. AR was defined as hepatectomy more than segmentectomy in Couinaud's classification [6] or the Brisbane 2000 Terminology [8], which is the same as subsegmentectomy in the Japanese general guidelines [7], and SAR was defined as hepatic resection of the small anatomical territory feeding from the 4th order portal vein branch or one of several 3rd order portal vein branches that flow into the same segment. The 47 patients in this study who underwent NAR were divided into PR $(n=25)$ and SAR $(n=22)$ groups (Fig. $1)$.

A history of the presenting illness was obtained for all patients and a complete physical examination was performed. A self-administered questionnaire was used to gain information on previous medical history. Excessive alcohol consumption was defined as an average daily consumption of an amount equivalent to $80 \mathrm{~g}$ of pure ethanol over a period of $>10$ years. Diabetes mellitus included patients whose blood sugar levels were controlled with medication. Patient infectious status for $\mathrm{HBV}$ and $\mathrm{HCV}$ (hepatitis $B$ and $C$ viruses) was determined by testing for hepatitis B surface antigen and hepatitis $C$ antibodies. Liver function was assessed by liver biochemistry testing, Child-Pugh score, ICG retention test (ICGR15), and technetium-99m-galactosyl human serum albumin scintigraphy $[9,10]$. Tumor progression and resectability were assessed with imaging studies, such as contrast enhanced computed tomography (CT), magnetic resonance imaging, and ultrasonography. Around 2006, 3D simulation software packages (Virtual Place, AZE Company, Japan; Synapse Vincent, Fujifilm Corporation, Japan) were introduced in our institution. The 3D images of the portal vein, hepatic vein, and tumor were also made preoperatively, based on multidetector CT, and segmentation or subsegmentation was performed. Informed consent of therapeutic protocols and use of clinicopathological data for this study were obtained from all patients.

\section{Surgical procedures}

The extent of liver resection was largely determined according to Makuuchi's criteria [11, 12] and the general condition of the patient. The resection method, namely, PR or SAR, was selected by considering the location and size of the tumor, the detectability of a portal vein 
branch on preoperative CT or reconstructed 3D images, and the tolerability of the effect on liver function for the calculated volume of the small anatomical territory including the tumor (Fig. 1). In the case of PR, a surgical margin of $5-10 \mathrm{~mm}$ from the tumor was secured, unless the tumor was attached to the hepatic vein or the glissonian pedicle to be preserved. In the case of SAR, the portal pedicle supplying the tumor is detected by preoperative CT and reconstructed $3 \mathrm{D}$ images. Then, by selecting the root of the 4th order portal vein branch or the root of one of several 3rd order branches that flow into the same segment, the small anatomical territory of the portal vein is extracted (Fig. 2). If part of the tumor is outside the extracted territories, another portal pedicle nearby is selected as a second target. After laparotomy or laparoscopic approach, the target portal branch was injected with 3 to $5 \mathrm{~mL}$ indigo carmine dye or $2.5 \mathrm{mg}$ of ICG (Daiichi Sankyo Company, Limited, Tokyo, Japan) under intraoperative US (Pro Focus Ultra View 2202, BK Medical, Denmark) guidance. When ICG was injected, the stained surface was identified using a near-infrared camera system (pde-neo, Hamamatsu Photonics K.K., Japan; IRI, Olympus Corporation, Japan) and marked using electrocautery as a transection line. The stained area was referred to the 3D image, and the anatomical relationship with the HCC also was verified. For hepatic parenchymal transection, we used an ultrasonic dissector (CUSA Excel $^{\mathrm{TM}}$; Integra Lifesciences Corporation, Plainsboro, NJ, USA) to identify intrahepatic structures such as hepatic veins and Glissons sheaths. Exposed structures of $2 \mathrm{~mm}$ or less were dissected using EnSeal $^{\mathrm{TM}}$ (Ethicon Endo-Surgery, Cincinnati, $\mathrm{OH}$, USA) and larger vessels were ligated with 3-0 braided silk or vessel clips. Pringle's maneuver (hepatic inflow occlusion time 15 minutes and reperfusion time 5 minutes) was performed during parenchymal transection.

\section{Patient follow-up}

The median follow-up period was 1332 days (range, 25 to 3120 days). Postoperative complications were investigated and graded according to the Clavien classification [13, 14]. In the present study, postoperative mortality was defined as all in-hospital deaths that occurred after surgery. Postoperative follow-up consisted of dynamic CT and laboratory tests, including measurement of the serum alpha-fetoprotein (AFP), AFP-L3, and des-gamma carboxyprothrombin (DCP) levels every 3-4 months during the first 5 years after surgery. Intrahepatic recurrence (IHR) pattern was classified into: (1) recurrence in the same Couinaud's segment as the initial tumor; (2) recurrence in a different segment from the initial tumor; (3) multisegmental recurrence. Any recurrence of disease was treated as vigorously as possible.
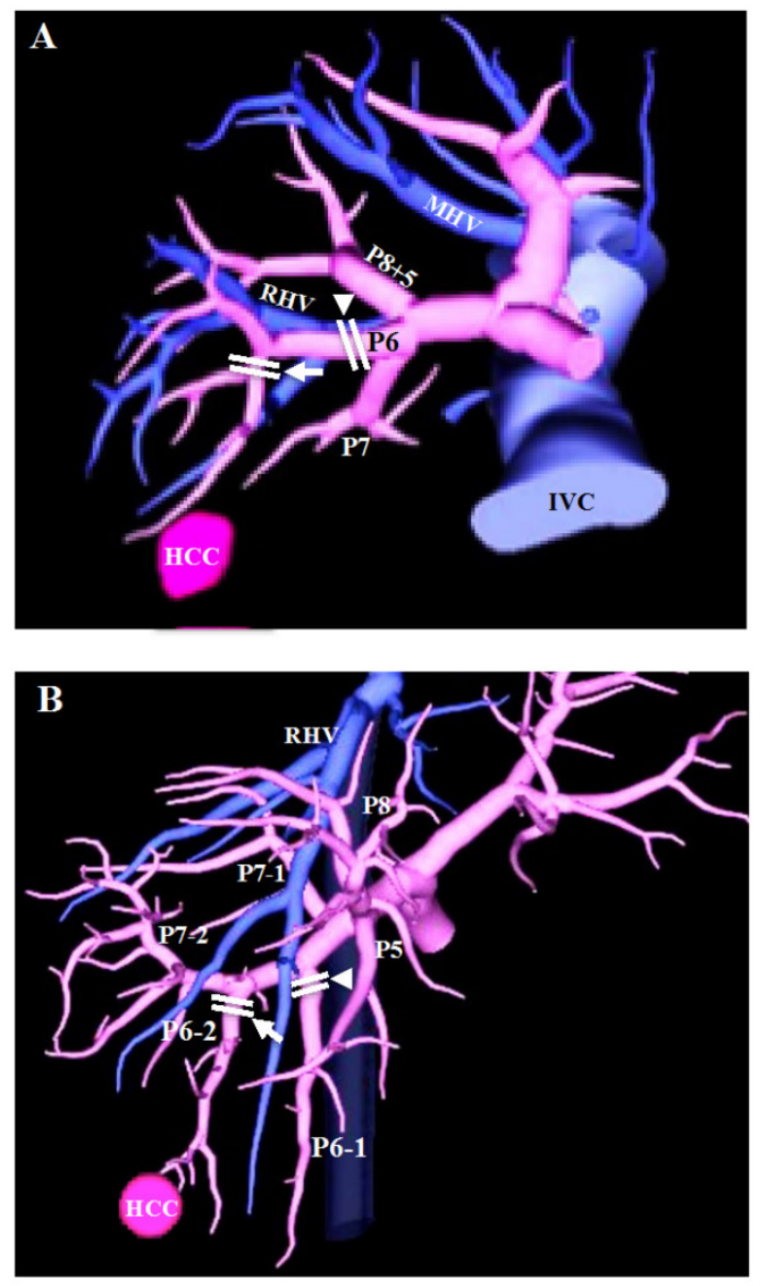

Figure 2. 3D images based on multidetector CT. (A) The 3rd order portal vein branch (arrowhead) for staining and dissection in Couinaud's segmentectomy, same as subsegmentectomy under Japanese general guidelines, and the 4th order portal vein branch (arrow) for staining and dissection in SAR of this study. (B) The 3rd order portal vein branches (arrowhead and arrow) for staining and dissection in Couinaud's segmentectomy, and one of the 3rd order portal vein branches (arrow) for staining and dissection in SAR of this study. PX: segmental portal vein branch of segment X; MHV: middle hepatic vein; RHV: right hepatic vein; IVC: inferior vena cava.

\section{Statistical analysis}

Differences between the two groups were analyzed by using the unpaired $t$ test for continuous variables and the $\chi^{2}$ test for categorical variables. The continuous data are presented as the mean plus range, except for AFP, DCP, operative time, and intraoperative blood loss. Recurrence-free and overall survival curves were calculated with the Kaplan-Meier method and compared by using the log-rank test. Risk factor analysis for recurrence was performed by the Cox proportional hazards 
regression test. The cutoff values for continuous data in univariate and multivariate analysis were defined as median values. Only significant variables in univariate analysis were included in the multivariate analysis. A $p$ value of less than 0.05 was considered to be significant.

\section{Results}

\section{Preoperative characteristics, surgical outcome, and tumor characteristics}

The preoperative characteristics of both groups are shown in Table 1 . There were no significant differences in the preoperative characteristics and the liver function variables, such as the ICGR15, Child-Pugh classification, or grade of liver damage between the two groups.

Table 2 shows the surgical outcome and tumor characteristics for both groups. The most common tumor locations were segment VI and VIII in the PR group, and were segment VI in the SAR group. Operative time was significantly longer in the SAR group than in the PR group. There was no significant difference in the postoperative morbidity between the two groups. However, one patient who underwent PR for a solitary HCC located at the border between segment IV and segment VIII died 39 days after the operation because of postoperative hemorrhage and liver failure. There were no significant differences in tumor size, gross classification, microscopic vascular invasion, or differentiation of the tumors between the two groups. The surgical margin was significantly smaller in the PR group than in the SAR group.

Table 1. Preoperative characteristics of the entire study population

\begin{tabular}{llll}
\hline Variables & $\begin{array}{l}\text { PR } \\
(n=25)\end{array}$ & $\begin{array}{l}\text { SAR } \\
(n=22)\end{array}$ & $p$ value \\
\hline Age (years) & $68(41-79)$ & $72(53-81)$ & 0.181 \\
Gender, Male / Female & $19 / 6$ & $12 / 10$ & 0.212 \\
BMI $\left(\mathrm{kg} / \mathrm{m}^{2}\right)$ & $24.0(18.4-31.8)$ & $23.6(19.7-27.8)$ & 0.674 \\
Diabetes mellitus, yes / no & $5 / 20$ & $7 / 15$ & 0.549 \\
Alcohol consumption, yes / no & $10 / 15$ & $3 / 19$ & 0.090 \\
HBsAg, positive / negative & $3 / 22$ & $4 / 18$ & 0.848 \\
Anti-HCV-Ab, positive /negative & $18 / 7$ & $10 / 12$ & 0.119 \\
Child-Pugh classification, A / B & $21 / 4$ & $22 / 0$ & 0.147 \\
Liver damagea , A / B & $19 / 6$ & $17 / 5$ & $>.9999$ \\
ICGR15 (\%) & $20.1(1.2-48)$ & $17.5(4.3-58)$ & 0.434 \\
Platelets $(x 104 / \mu L)$ & $13.4(5.4-25.3)$ & $14.6(6.3-31.7)$ & 0.559 \\
Prothrombin time (\%) & $91(60-116)$ & $95(86-106)$ & 0.150 \\
Albimin $(\mathrm{g} / \mathrm{dL})$ & $3.9(3.0-4.8)$ & $3.8(2.5-4.5)$ & 0.394 \\
Total bilirubin (mg/dL ) & $0.9(0.4-1.6)$ & $0.8(0.3-1.7)$ & 0.286 \\
AST (IU/L) & $47(21-97)$ & $38(21-75)$ & 0.075 \\
ALT (IU/L) & $45(13-89)$ & $35(11-114)$ & 0.130 \\
AFP (ng/ml) ${ }^{\mathrm{b}}$ & $14(2-1087)$ & $8(2.2-37537)$ & 0.277 \\
DCP (mAU/ml) & $29(11-7849)$ & $24(11-1951)$ & 0.594 \\
\hline
\end{tabular}

a According to the criteria of the Liver Cancer study Group of Japan [ref. 7]

b Median (range)

BMI: body mass index; ICGR15: indocyanine green retention rate at $15 \mathrm{~min}$.
Table 2. Perioperative variables and tumor factors

\begin{tabular}{|c|c|c|c|}
\hline Variables & $\begin{array}{l}\mathrm{PR} \\
(n=25)\end{array}$ & $\begin{array}{l}\text { SAR } \\
(n=22)\end{array}$ & $p$ value \\
\hline \multicolumn{4}{|l|}{ Operative variables } \\
\hline \multicolumn{4}{|l|}{ Main location of the tumor, no. } \\
\hline $\begin{array}{l}\text { Segment } \\
\text { I/II/III/IV/V/VI/VII/VIII }\end{array}$ & $\begin{array}{l}0 / 1 / 2 / 2 / 5 / 7 \\
/ 1 / 7\end{array}$ & $\begin{array}{l}0 / 0 / 1 / 0 / 5 / 12 \\
/ 2 / 2\end{array}$ & 0.269 \\
\hline Operative time (min)a & $255(141-498)$ & $312(228-619)$ & 0.009 \\
\hline Intraoperative blood loss $(\mathrm{ml})^{\mathrm{a}}$ & $220(15-3425)$ & $720(5-2480)$ & 0.298 \\
\hline \multicolumn{4}{|l|}{ Postoperative morbidity } \\
\hline Clavien grade 1 or more, no. (\%) & $7(28)$ & $7(32)$ & $>.9999$ \\
\hline Clavien grade 3 or more, no. (\%) & $2(8)$ & $2(9.1)$ & $>.9999$ \\
\hline Mortality, no. (\%) & $1(4)$ & $0(0)$ & $>.9999$ \\
\hline $\begin{array}{l}\text { Postoperative hospitalization } \\
\text { (days) }\end{array}$ & $14(6-39)$ & $13(9-24)$ & 0.561 \\
\hline \multicolumn{4}{|l|}{ Tumor factors } \\
\hline Size $(\mathrm{mm})$ & $22(8-50)$ & $25(10-45)$ & 0.349 \\
\hline \multicolumn{4}{|l|}{ Gross classification } \\
\hline $\begin{array}{l}\text { Boundary type }{ }^{b} / \text { Non-boundary } \\
\text { typec }\end{array}$ & $19 / 6$ & $18 / 4$ & 0.892 \\
\hline $\begin{array}{l}\text { Microscopic pv invasion positive, } \\
\text { no. }(\%)\end{array}$ & $2(8)$ & $2(9.1)$ & $>.9999$ \\
\hline $\begin{array}{l}\text { Microscopic hv invasion positive, } \\
\text { no. }(\%)\end{array}$ & $1(4)$ & $1(4.5)$ & $>.9999$ \\
\hline $\begin{array}{l}\text { Tumor differentiation } \\
\text { well / moderately / poorly }\end{array}$ & $6 / 17 / 2$ & $10 / 10 / 2$ & 0.268 \\
\hline Surgical margin (mm) & $4.0(0-14)$ & $9.8(1.0-35)$ & 0.001 \\
\hline TNM staged, I / II / III & $11 / 11 / 3$ & $8 / 10 / 4$ & 0.789 \\
\hline \multicolumn{4}{|c|}{ a Median (range) } \\
\hline \multicolumn{4}{|c|}{$\begin{array}{l}\text { b Boundary type: small nodular type with indistinct margin and simple nodular } \\
\text { type }\end{array}$} \\
\hline \multicolumn{4}{|c|}{$\begin{array}{l}\text { d According to the criteria of the Liver Cancer study Group of Japan [ref. 7] } \\
\text { pv: portal vein; hv: hepatic vein. }\end{array}$} \\
\hline
\end{tabular}

\section{Postoperative RFS and overall survival}

The cumulative 1-, 3-, and 5-year RFS rates were 87.3, 26.8, and $26.8 \%$ in the PR group, and 88.5, 75.4 and $67.8 \%$ in the SAR group, respectively (Fig. 3A). The cumulative 1-, 3-, and 5-year overall survival rates were 92.0, 82.0, and $69.8 \%$ in the PR group, and $100,92.9$, and $92.9 \%$ in the SAR group, respectively (Fig. 3B). There was a significant difference in the recurrence-free survival rates between the two groups.

\section{The patterns of recurrence after surgery}

The patterns of recurrence are shown in Table 3. The rate of recurrence was significantly higher in the PR group than in the SAR group $(P=0.002)$. There was no significant difference in the number of tumor recurrences. The sites of extrahepatic recurrence (EHR) were lung in the PR group, and local dissemination in the SAR group. IHR in the SAR group was significantly less than in the PR group. Although there was no significant difference in the pattern of IHR between the two groups, the IHR in the same segment as the initial tumor tended to be greater in the PR group (12\%) than in the SAR group (4.5\%). The locations of recurrence in the same segment as the initial tumor were dispersed. 

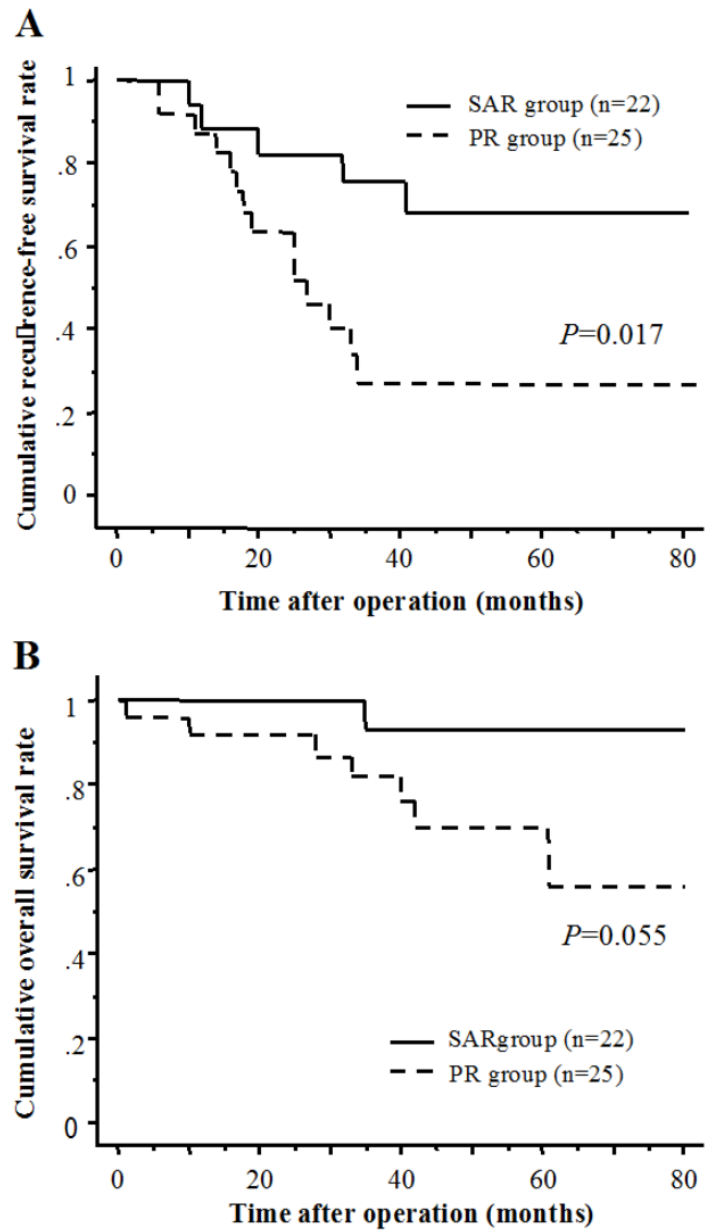

Figure 3. (A) Recurrence-free survival of $S A R$ group and $P R$ group. (B) Cumulative overall survival of SAR group and PR group.

Table 3. Recurrence patterns of HCC in PR and SAR

\begin{tabular}{llll}
\hline Variables & $\begin{array}{l}\text { PR } \\
(n=25)\end{array}$ & $\begin{array}{l}\text { SAR } \\
(n=22)\end{array}$ & $\begin{array}{c}p \\
\text { value }\end{array}$ \\
\hline $\begin{array}{l}\text { Number of recurrence tumor, no. (\%) } \\
\text { Solitary }\end{array}$ & $\begin{array}{l}7(28.0) \\
7(28.0)\end{array}$ & $\begin{array}{l}1(4.5) \\
4(18.2)\end{array}$ & 0.505 \\
$\begin{array}{l}\text { Multiple } \\
\text { Site of recurrence, no. (\%) }\end{array}$ & $1(4.0)$ & $1(4.5)$ & $>.9999$ \\
$\begin{array}{l}\text { Extrahepatic recurrence } \\
\text { Intrahepatic recurrence }\end{array}$ & $14(56.0)$ & $4(18.2)$ & 0.018 \\
$\begin{array}{l}\text { Pattern of intrahepatic recurrence, no. } \\
\text { (\%) }\end{array}$ & & & 0.455 \\
$\begin{array}{l}\text { In the same segment as the initial tumor } \\
\text { In a different segment from the initial }\end{array}$ & $3(12.0)$ & $1(4.5)$ & \\
$\begin{array}{l}\text { tumor } \\
\text { Multisegmental recurrence }\end{array}$ & $3(12.0)$ & $2(9.1)$ & \\
$\begin{array}{l}\text { Location of the recurrence in the same } \\
\text { segment }\end{array}$ & & & \\
$\begin{array}{l}\text { as the initial tumor, no. } \\
\text { Segment V / VI / VII / VIII }\end{array}$ & $1 / 1 / 0 / 10 / 1 / 0 / 0$ & $>.9999$ \\
\hline
\end{tabular}

\section{Risk factors for recurrence after operation by univariate and multivariate analyses.}

The results using the Cox hazards regression test for risk factors of recurrence are shown in Table 4. In the univariate analysis, positivity for hepatitis $C$ virus antibodies, AST $\geq 38 \mathrm{IU} / \mathrm{L}, \mathrm{ALT} \geq 32 \mathrm{IU} / \mathrm{L}, \mathrm{AFP}$ $\geq 10 \mathrm{ng} / \mathrm{ml}$, and PR were significant factors for a poorer RFS rate. The multivariate analysis revealed that the PR operative procedure remained as a significant independent risk factor for poorer RFS.

Table 4. Risk factors for RFS after operation by univariate and multivariate analyses

\begin{tabular}{|c|c|c|c|c|}
\hline \multirow[t]{2}{*}{ Variables } & \multicolumn{2}{|l|}{ Univariate } & \multicolumn{2}{|l|}{ Multivariate } \\
\hline & $\begin{array}{l}\text { Hazard ratio } \\
(95 \% \mathrm{CI})\end{array}$ & $\mathrm{P}$ & $\begin{array}{l}\text { Hazard ratio } \\
(95 \% \mathrm{CI})\end{array}$ & $\mathrm{P}$ \\
\hline Age ( $\geq 70$ years $)$ & $1.56(0.61-3.97)$ & 0.355 & & \\
\hline Gender (male) & $0.92(0.36-2.36)$ & 0.868 & & \\
\hline $\mathrm{BMI}\left(\geq 24 \mathrm{~kg} / \mathrm{m}^{2}\right)$ & $0.96(0.39-2.36)$ & 0.923 & & \\
\hline Diabetes mellitus (yes) & $1.20(0.40-3.61)$ & 0.752 & & \\
\hline $\begin{array}{l}\text { Alcohol consumption } \\
\text { (yes) }\end{array}$ & $2.37(0.92-6.10)$ & 0.074 & & \\
\hline HBsAg (positive) & $0.20(0.03-1.51)$ & 0.119 & & \\
\hline Anti-HCV-Ab (positive) & $4.27(1.24-14.7)$ & 0.022 & $2.42(0.54-10.75)$ & 0.248 \\
\hline $\begin{array}{l}\text { Child-Pugh classification } \\
\text { (B) }\end{array}$ & $2.54(0.71-9.09)$ & 0.152 & & \\
\hline Liver damagea $(\mathrm{B})$ & $2.16(0.81-5.71)$ & 0.124 & & \\
\hline ICGR15 ( $\geq 18 \%)$ & $2.26(0.85-5.96)$ & 0.101 & & \\
\hline Platelets $\left(<12 \times 10^{4} / \mu \mathrm{L}\right)$ & $2.34(0.89-6.17)$ & 0.085 & & \\
\hline $\begin{array}{l}\text { Prothrombin time }(<90 \\
\%)\end{array}$ & $2.05(0.83-5.08)$ & 0.119 & & \\
\hline Albimin $(<4.0 \mathrm{~g} / \mathrm{dL})$ & $1.89(0.74-4.85)$ & 0.184 & & \\
\hline $\begin{array}{l}\text { Total bilirubin }(\geq 0.8 \\
\mathrm{mg} / \mathrm{dL})\end{array}$ & $1.07(0.38-2.98)$ & 0.897 & & \\
\hline AST $(\geq 38 \mathrm{IU} / \mathrm{L})$ & $3.64(1.30-10.2)$ & 0.014 & $1.82(0.37-8.90)$ & 0.459 \\
\hline $\operatorname{ALT}(\geq 32 \mathrm{IU} / \mathrm{L})$ & $2.94(1.15-7.53)$ & 0.025 & $0.84(0.19-3.70)$ & 0.817 \\
\hline $\operatorname{AFP}(\geq 10 \mathrm{ng} / \mathrm{ml})$ & $3.01(1.16-7.80)$ & 0.024 & $1.93(0.60-6.17)$ & 0.268 \\
\hline $\mathrm{DCP}(\geq 27 \mathrm{mAU} / \mathrm{ml})$ & $2.21(0.84-5.78)$ & 0.107 & & \\
\hline Operative procedure (PR) & $3.32(1.17-9.43)$ & 0.024 & $3.36(1.10-10.20)$ & 0.033 \\
\hline $\begin{array}{l}\text { Operative time }(\geq 280 \\
\text { min) }\end{array}$ & $0.81(0.33-2.00)$ & 0.647 & & \\
\hline $\begin{array}{l}\text { Intraoperative blood loss } \\
(\geq 450 \mathrm{ml})\end{array}$ & $0.65(0.25-1.64)$ & 0.359 & & \\
\hline Surgical margin $(<5 \mathrm{~mm})$ & $1.76(0.71-4.33)$ & 0.222 & & \\
\hline $\begin{array}{l}\text { Postoperative morbidity } \\
\text { (Clavien grade } 3 \text { or } \\
\text { more) }\end{array}$ & $0.78(0.10-5.85)$ & 0.809 & & \\
\hline Tumor size $(\geq 2.0 \mathrm{~cm})$ & $0.92(0.36-2.35)$ & 0.860 & & \\
\hline $\begin{array}{l}\text { Gross classification } \\
\text { (Non-boundary typeb) }^{\text {Non- }}\end{array}$ & $0.84(0.25-2.90)$ & 0.788 & & \\
\hline $\begin{array}{l}\text { Microscopic pv invasion } \\
\text { (positive) }\end{array}$ & $1.69(0.38-7.46)$ & 0.490 & & \\
\hline $\begin{array}{l}\text { Microscopic hv invasion } \\
\text { (positive) }\end{array}$ & $2.97(0.65-13.5)$ & 0.158 & & \\
\hline $\begin{array}{l}\text { Tumor differentiation } \\
\text { (poorly) }\end{array}$ & $2.24(0.29-17.2)$ & 0.440 & & \\
\hline TNM stage $(\mathrm{III})$ & $1.18(0.34-4.06)$ & 0.799 & & \\
\hline
\end{tabular}

\section{Discussion}

The beneficial effects of AR for HCC have been shown in terms of postoperative survival and disease-free survival [1-4]. In these reports, it is reported that vascular invasion of tumor and intrahepatic metastasis were among the risk factors that most strongly influenced the postoperative 
prognosis, and AR was recommended as a procedure for efficient eradication of minute cancerous foci with a main tumor and intrahepatic metastasis. We also reported that AR of a single HCC less than $3 \mathrm{~cm}$ in diameter was superior to NAR or ablation therapies with regard to overall and disease-free survival, and the effect of AR was more prominent in the subgroup of HCCs with extranodular growth [5]. On the other hand, some authors have not been able to demonstrate significant survival benefits for $A R$ [15-17]. In these reports, it is reported that the postoperative recurrence patterns were similar in the NAR and AR groups, and neither NAR nor AR can prevent multicentric recurrence or carcinogenesis. Thus, the debate surrounding anatomical versus non-anatomical resection remains controversial. In the present study, we retrospectively compared the RFS for the PR group and for the SAR group, which is regarded as NAR for a solitary HCC of $5 \mathrm{~cm}$ or less with impaired liver function, and evaluated the impact of SAR on recurrence after curative surgery.

HCC has a tendency to metastasize via the portal vein, and the resection of liver parenchyma fed by portal venous branches bearing the tumor is considered to be a logical method for eliminating potential intrahepatic metastases [18]. Therefore, we thought that AR should be considered for improving surgical outcome, and AR was performed for primary HCC, where possible. However, NAR such as partial resection and enucleation was also selected often by considering patient background variables, including comorbidity, the degree of impairment of liver function and tumor factors. Anatomical segmentectomy and subsegmentectomy have been proposed as a means of improving curative surgical treatment for HCC and preserving liver parenchyma in patients with impaired liver function $[2,19]$. In a typical anatomical resection method such as segmentectomy, the surface of the segment area is marked according to the area stained after injection of blue dye under intraoperative US guidance [19, 20]. Aiming at improvement of prognosis with NAR, we have aggressively employed resection of small anatomical divisions as an alternative to PR for patients with impaired liver function; the method employs identification of the 3rd or 4 th order portal vein territory feeding the domain using dye injection under intraoperative US guidance.

In this study, the resection method was selected by considering the detectability of a portal vein branch and the tolerability of the effect on liver function for the calculated volume of the small anatomical territory including the tumor on preoperative images. Therefore there was a possibility that selection bias existed between the two groups, but there were no significant differences between groups in the preoperative characteristics and the liver function variables, such as the ICGR15, Child-Pugh classification, or grade of liver damage.

The most tumor common locations in the PR group were segment VI and VIII. Although segment VIII resection or the resection of the ventral or dorsal portion, i.e., subsegmentectomy can be anatomically performed using a staining method and full exposure of the landmark vessels [2, 19-21], the volume of segment VIII calculated by 3D perfusion-based volumetry represented the largest proportion (11.1-38.0\%) of total liver volume [22]. Thus, it seems that the volume of $A R$ and the complexity of the operative procedure became problematic for patients with impaired liver function; consequently, PR was often selected in segment VIII. The branching variations of the portal vein in segment V (P5) and VI (P6) were complicated. The number of P5s ranged from one to six, and the branching of P6 was classified into a bifurcation type, a direct bifurcation type from the right portal vein, and a bow-shaped type [22]. The staining of portal vein territory under intraoperative US guidance in segment V or VI was relatively easily achieved. Therefore, many tumors in segment VI and $\mathrm{V}$ underwent SAR in this study group. In this area, laparoscopic or laparoscopic-assisted hepatectomy seems easily compared with upper segment, e.g., segment VII and VIII; we had reported the value of pure laparoscopic or laparoscopic-assisted SAR using puncture of the portal vein branch with dye injection under US guidance [23-25].

For precise puncture of the target portal vein, it is important to establish the anatomical relationship between the HCC and the surrounding structures preoperatively. Preoperative simulation using 3D images based on multidetector CT is useful for identifying the anatomical relationship between the tumor and the portal branch and comparing the intraoperative staining area with the planned resection area [23-25]. Mise et al. reported that Couinaud's eight segments vary significantly in territory and volume, and have variable patterns of portal vein branching. Therefore, perfusion-based 3D is a useful tool for preoperative evaluation when planning anatomic segmentectomy [22]. 3D simulation appears essential for identification of the portal vein territory and accurate anatomical resection.

The present study revealed that SAR was superior to PR in terms of recurrence-free survival in patients with a solitary HCC of $5 \mathrm{~cm}$ or less. There were no significant differences in the number of tumor recurrences and the rate of EHR. Previous studies reported that EHR after curative hepatectomy 
occurred in $7.2-15.5 \%$ of patients [26-30], and the incidence of EHR was similar between the AR and the NAR group $[29,30]$. In this study, EHR was found in one patient $(4 \%)$ of the PR group concomitant with IHR, and in one patient $(4.5 \%)$ of the SAR group without IHR. In fact, $14.4 \%$ of the 111 patients with a primary and solitary HCC who underwent AR in our department developed EHR. Several studies reported that significant independent risk factors for EHR included microscopic hepatic vein invasion [26, 27], microscopic portal vein invasion [28], intraoperative blood loss [26] and tumor invading the capsule [27]. The patient with EHR in the PR group had microscopic portal vein invasion, and the patient with EHR in the SAR group had hepatic vein invasion. All initial tumors of two patients with EHR showed tumor capsule invasion. Intraoperative blood loss in two patients $(815 \mathrm{ml}$ and $1120 \mathrm{ml})$ was also greater than the median value for all patients in this study. Several studies reported that hematogeneous dissemination was the main presumed mechanism of extrahepatic spread [26, 27, 31]. Thus, it seems that there is always a chance that malignant cells may spread systemically in patients with vascular invasion, and the selection of the surgical method may not influence the incidence of EHR.

IHR is the most frequent mode of HCC recurrence; the IHR pattern in this study was classified into (1) recurrence in the same Couinaud's segment as the initial tumor; (2) recurrence in a different segment from the initial tumor; and (3) multisegmental recurrence. Although there was no significant difference in the pattern of IHR between the PR group and the SAR group, the IHR in the same segment as the initial tumor, and in the different segment from the initial tumor tended to be greater in the PR group than in the SAR group. One of the major forms of IHR is metastasis via vascular invasion, because HCC has a high propensity to invade the portal and hepatic veins [18]. Therefore, the removal of the area fed by the portal venous branch bearing the tumor and the securing of the surgical margin as long as possible should be recommended, even if liver function is impaired. On the other hand, multicentric carcinogenesis, which causes recurrence in different or multiple-segments, is considered to be uncontrollable by surgery, even by $\operatorname{AR}[29,30]$. In this study, there is a possibility that multicentric carcinogenesis in the PR group increased because of impaired liver function and the rate of patients who were anti-HCV-Ab positive (no significant differences between groups), and influenced the RFS rate. However, there is also the possibility that SAR reduced the occult tumor cells around the initial tumor, and consequently the IHR in the SAR group was decreased compared with PR.

In this study, positivity for hepatitis $C$ virus antibodies, AST, ALT, AFP, and PR were identified as risk factors for recurrence after curative hepatectomy by univariate analysis, and PR was identified as independent risk factor on multivariate analysis. Although the SAR in this study was smaller than anatomical segmentectomy or subsegmentectomy, and is regarded as a NAR, we think that SAR can in fact be regarded as an AR, because of removal of the portal vein territory.

The present study was associated with some limitations. First, the total number of patients was small in both groups. The aims of the present study were to compare recurrence after operation for primary and solitary $\mathrm{HCC}$ of $5 \mathrm{~cm}$ or less with impaired liver function, so the numbers of each group were relatively small. Additionally, this was a retrospective, nonrandomized, observational study; therefore, the rates of patients with excessive alcohol consumption, with anti-HCV-Ab positive or with Child-Pugh B, and the levels of AST and ALT tended to be higher in the PR group than in the SAR group $(p<0.2)$. Although further prospective studies are required to elucidate the true benefit of SAR, it seems that it is difficult to make clinical background, tumor factors, and strategy of operation even for prospective study in patients with impaired liver function.

In conclusion, SAR is effective in improving the rate of RFS after initial and curative resection in cases of primary and solitary HCC of $5 \mathrm{~cm}$ or less with impaired liver function compared with PR. Thus, SAR is considered to be a logical method for removal of the portal vein territory and a useful procedure for increasing the curative success rate.

\section{Competing Interests}

The authors have declared that no competing interest exists.

\section{References}

1. Kosuge $\mathrm{T}$, Makuuchi M, Takayama $\mathrm{T}$, et al. Long-term results after resection of hepatocellular carcinoma: experience of 480 cases. Hepatogastroenterology. 1993; 40: 328-332.

2. Hasegawa K, Kokudo N, Imamura H, et al. Prognostic impact of anatomic resection for hepatocellular carcinoma. Ann Surg. 2005; 242: 252-259.

3. Yamamoto M, Takasaki K, Ohtsubo T, et al. Effectiveness of systematized hepatectomy with Glisson's pedicle transection at the hepatic hilus for small nodular hepatocellular carcinoma: retrospective analysis. Surgery. 2001; 130: 443-448.

4. Eguchi S, Kanematsu T, Arii S, et al. Comparison of the outcomes between an anatomical subsegmentectomy and a non-anatomical minor hepatectomy for single hepatocellular carcinomas based on a Japanese nationwide survey. Surgery. 2008; 143: 469-475.

5. Ueno S, Kubo F, Sakoda M, et al. Efficacy of anatomic resection vs nonanatomic resection for small nodular hepatocellular carcinoma based on gross classification. J Hepatobiliary Pancreat Surg. 2008; 15: 493-500.

6. Couinaud C. Surgical Anatomy of the Liver Revised. Paris: Acheve D'imprimer Sur Les Presses; 1989. 
7. Liver Cancer Study Group of Japan. The general rules for the clinical and pathological study of primary liver cancer. 5th edition, revised version. 2009: (Tokyo).

8. Belgihiti J, Clavien PA, Gadzijev et al. The Brisbane 2000 terminology of liver anatomy and resections. HPB. 2000; 2: 333-339.

9. Fukui H, Kashiwagi T, Kasahara A, et al. Evaluation of usefulness of Tc-99m-GSA liver scintigraphy in chronic liver diseases. Kaku Igaku. 1991; 28: 1333-1341.

10. Torizuka K, Ha-Kawa SK, Kudo M, et al. Phase III multi-center clinical study on 99mTc-GSA, a new agent for functional imaging of the liver. Kaku Igaku. 1992; 29: 159-181.

11. Makuuchi M, Kosuge T, Takayama $\mathrm{T}$, et al. Surgery for small liver cancers. Semin Surg Oncol. 1993; 9: 298-304.

12. Imamura $\mathrm{H}$, Seyama $\mathrm{Y}, \mathrm{Kokudo} \mathrm{N}$, et al. One thousand fifty-six hepatectomies without mortality in 8 years. Arch Surg. 2003; 138: 1198-1206.

13. Dindo D, Demartines N, Clavien PA. Classification of surgical complications: a new proposal with evaluation in a cohort of 6336 patients and results of a survey. Ann Surg. 2004; 240: 205-213.

14. Clavien PA, Barkun J, de Oliveira ML, et al. The Clavien-Dindo classification of surgical complications: five-year experience. Ann Surg. 2009; 250: 187-196.

15. Kaibori M, Matsui Y, Hijikawa T, et al. Comparison of limited and anatomic hepatic resection for hepatocellular carcinoma with hepatitis C. Surgery. 2006; 139: 385-394.

16. Tanaka $\mathrm{K}$, Shimada $\mathrm{H}$, Matsumoto $\mathrm{C}$, et al. Anatomic versus limited nonanatomic resection for solitary hepatocellular carcinoma. Surgery. 2008; 143: 607-615.

17. Hirokawa F, Kubo S, Nagano H, et al. Do patients with small solitary hepatocellular carcinomas without macroscopically vascular invasion require anatomic resection? Propensity score analysis. Surgery. 2015; 157: 27-36.

18. Nakashima T, Kojiro M. Pathologic characteristics of hepatocellular carcinoma. Semin Liver Dis. 1986; 6: 259-266.

19. Makuuchi M, Hasegawa $H$, Yamazaki S. Ultrasonically guided subsegmentectomy. Surg Gynecol Obstet. 1985; 161: 346-350.

20. Takayama T, Makuuchi M, Watanabe K, et al. A new method for mapping hepatic subsegment: counterstaining identification technique. Surgery. 1991; 109: 226-229.

21. Takamoto T, Hashimoto T, Ogata $S$, et al. Planning of anatomical liver segmentectomy and subsegmentectomy with 3-dimensional simulation software. Am J Surg. 2013; 206: 530-538.

22. Mise Y, Satou S, Shindoh J, et al. Three-dimensional volumetry in 107 normal livers reveals clinically relevant inter-segment variation in size. HPB (Oxford). 2014; 16: 439-447.

23. Ueno S, Sakoda M, Kurahara H, et al. Preoperative segmentation of the liver, based on 3D CT images, facilitates laparoscopic anatomic hepatic resection for small nodular hepatocellular carcinoma in patients with cirrhosis. Hepato-Gastroenterology. 2010; 57: 807-812.

24. Sakoda M, Ueno S, Iino S, et al. Pure laparoscopic subsegmentectomy of the liver using a puncture method for the target portal branch under percutaneous ultrasound with artificial ascites. Surg Laparosc Endosc Percutan Tech. 2013; 23: e45-e48.

25. Sakoda M, Ueno S, lino S, et al. Anatomical laparoscopic hepatectomy for hepatocellular carcinoma using indocyanine green fluorescence imaging. J Laparoendosc Adv Surg Tech A. 2014; 24: 878-882.

26. Taketomi A, Toshima T, Kitagawa D, et al. Predictors of extrahepatic recurrence after curative hepatectomy for hepatocellular carcinoma. Ann Surg Oncol. 2010; 17: 2740-2746.

27. Tanaka K, Shimada H, Matsuo K, et al. Clinical features of hepatocellular carcinoma developing extrahepatic recurrences after curative resection. World J Surg. 2008; 32: 1738-1747.

28. Hirokawa F, Hayashi M, Miyamoto Y, et al. Surgical treatment of extrahepatic recurrence of hepatocellular carcinoma. Langenbecks Arch Surg. 2014; 399: 1057-1064.

29. Okamura $\mathrm{Y}$, Ito $\mathrm{T}$, Sugiura $\mathrm{T}$, et al. Anatomic versus nonanatomic hepatectomy for a solitary hepatocellular carcinoma : a case-controlled study with propensity score matching. J Gastrointest Surg. 2014; 18: 1994-2002.

30. Marubashi S, Gotoh K, Akita H, et al. Analysis of Recurrence Patterns After Anatomical or Non-anatomical Resection for Hepatocellular Carcinoma. Ann Surg Oncol. 2015; 22: 2243-2252.

31. Kim PN, Kim IY, Lee KS, et al. Intraperitoneal seeding from hepatoma. Abdom Imaging. 1994; 19: 309-312. 